Nonlinear Processes in Geophysics, 12, 755-765, 2005

SRef-ID: $1607-7946 / \mathrm{npg} / 2005-12-755$

European Geosciences Union

(C) 2005 Author(s). This work is licensed

under a Creative Commons License.

\title{
Comparison of extended and ensemble based Kalman filters with low and high resolution primitive equation ocean models
}

\author{
I. Hoteit ${ }^{1}$, G. Korres ${ }^{2}$, and G. Triantafyllou ${ }^{2}$ \\ ${ }^{1}$ Scripps Institution of Oceanography, La Jolla, CA, USA \\ ${ }^{2}$ Hellenic Center for Marine Research, Anavissos, Greece \\ Received: 20 September 2004 - Revised: 26 May 2004 - Accepted: 21 June 2005 - Published: 2 August 2005 \\ Part of Special Issue "Quantifying predictability"
}

\begin{abstract}
Kalman filters are widely used for data assimilation into ocean models. The aim of this study is to discuss the relevance of these filters with high resolution ocean models. This was investigated through the comparison of two advanced Kalman filters: the singular evolutive extended Kalman (SEEK) filter and its ensemble-based variant, called SEIK filter. The two filters were implemented with the Princeton Ocean model (POM) considering a low spatial resolution configuration (Mediterranean sea model) and a very high one (Pagasitikos Gulf coastal model). It is shown that the two filters perform reasonably well when applied with the low resolution model. However, when the high resolution model is considered, the behavior of the SEEK filter seriously degrades because of strong model nonlinearities while the SEIK filter remains remarkably more stable. Based on the assumption of prior Gaussian distributions, the linear analysis step of the latter can still be improved though.
\end{abstract}

\section{Introduction}

The recent dramatical progress in computing power enabled the use of very high resolution numerical models to simulate the general circulation of different oceanic areas while continuously incorporating more fine scale information. Although these highly sophisticated models are now capable of adequately reconstructing most of the variability of the studied areas, different sources of errors, related to modeling approximations and/or uncertainties in the model parameters, might strongly degrade their performance. These errors cause very often a large drift of the model trajectory from reality, if the models are not somehow constrained with real ocean measurements. The latter framework is called data assimilation and it was shown to be the best way to improve consistency between ocean models and observations. Following meteorology, assimilation techniques are

Correspondence to: G. Triantafyllou

(gt@ncmr.gr) now widely used in oceanography. They can be classified into two classes: sequential approach and variational approach (Ghil and Malanotte-Rizzoli, 1991). Sequential methods proceed by incrementally correcting the discrepancy between observations and a model prediction based on prior information about uncertainties in the model and data. Variational methods seek to minimize the misfit between data and model trajectory over a given period of time through the adjustments of a well chosen set of control parameters.

Using simple dynamical models, several studies demonstrated that the application of the current data assimilation techniques can be problematic when these models are highly nonlinear (Evensen, 1994; Miller et al., 1994). This is expected to be also true for high resolution ocean models since fine scale variations are naturally associated with strong model nonlinearities. For instance, in variational methods the cost function becomes highly nonlinear preventing any improvement with an affordable optimization algorithm (Köhl and Willebrand, 2002). Sequential methods are generally based on the Kalman filter which is only optimal for linear models. Until recently, the practice was to linearize the model about the most recent estimate, leading to the socalled extended Kalman (EK) filter (Jazwinski, 1970). This approximation might however provoke the divergence of the filter when used with a strongly nonlinear model (Evensen, 1992; Gauthier et al., 1993). Nonlinear ensemble techniques based on Monte-Carlo methods were therefore developed to avoid the linearization of the model (Evensen, 1994).

A comparison of the extended and the ensemble approaches cannot be assessed a priori for a particular nonlinear model but must be determined by simulations (Jazwinski, 1970). Because of significant computing requirements, simple dynamical models, as the Lorenz model, were so far used to compare the two approaches (Evensen, 1992; Miller et al., 1994). However, these works pointed out the need of better understanding the behavior of these methods in more realistic frameworks. Madsen and Canizares (1999) used a hypothetical bay region model and concluded that the performance of both approaches is comparable. Similar conclusions were 
also made by Verlaan and Heemink (1995) and Nerger et al. (2005) using simple ocean models. All these previous studies were, however, based on weakly nonlinear models. This paper considers this problem through the implementation of the singular evolutive extended Kalman (SEEK) filter, which is a suboptimal EK filter, and of its ensemble-based variant, called SEIK filter, with two different configurations of the Princeton Ocean model (POM) using a low spatial resolution $\left(1 / 4^{\circ} \times 1 / 4^{\circ}\right)$ for the whole Mediterranean Sea and a very high one $\left(1 / 100^{\circ} \times 1 / 100^{\circ}\right)$ for the Pagasitikos Gulf. The application of these two filters with fully realistic ocean models was possible because of the use of low rank error covariance matrices, making the computations relatively affordable.

Recently, a similar work was presented by Zang and Malanotte-Rizzoli (2003) using a simple quasi-geostrophic model with two different eddy viscosity parameterization cases (low and high) and concluded that the ensemble method generally behaves better. However, the comparison of the two approaches was carried out between an ensemble Kalman filter and a steady-state simplified EK filter, where the evolution of the filter's error covariance matrix was not carried out and this may result in a significant degradation of the EK filters performances (Hoteit and Pham, 2003). This approach made difficult the interpretation of their results since it was quite unclear how to separate and/or quantify the errors due to the linearization from the errors induced by the use of time-invariant error covariance matrices. The SEEK and SEIK filters used in the present study are "more comparable" by construction since they are based on the same assumption. The only difference between them lies in the use of linearization (SEEK) or Monte-Carlo techniques (SEIK) for the evolution of the error covariance matrices, making the comparison more straightforward. Numerical experiments are based on a twin experiments approach which provides an efficient way for an inter-comparison between the performances of two assimilation techniques since all uncertain inputs are known by design. This is very convenient for the present study because it enables to clearly and easily identify which filter provides the best estimate for all the state variables. Additionally, it eliminates the influence of different filters' parameters, especially the model error, allowing to attribute the differences between the filters' solutions only to the method used for the evolution of the filters statistics. The result of an experiment assimilating AVISO sea level anomaly data into POM for the Mediterranean Sea configuration is also presented and discussed to show that the conclusions from the former framework still hold in a different setup where the influence of the model error is much more pronounced.

Section 2 briefly summarizes the SEEK and SEIK filters. The ocean models are described in Sect. 3. The design and the results of the numerical experiments are presented in Sect. 4. Finally, a general discussion concludes the paper in Sect. 5.

\section{The filters}

The Kalman filter is a data assimilation technique that recursively generates an optimal analysis, in the least-square sense, of the state of a linear system given a set of measurements (Kalman, 1960). It operates in two steps starting from an initial estimate of the state and the corresponding error covariance matrix: (i) forecast step using the model, and (ii) analysis step to correct the forecast each time new observations are available. The filter is very powerful in several aspects: it supports estimations of past, present, and even future system states, and it can do so even when the model and the measurements are noisy. The filter however requires that the system and measurement noises are uncorrelated, additive, white and Gaussian. Indeed, linear models interact uniquely well with Gaussian noise since under this assumption, the filter's distributions are Gaussian and the calculations are easy. Note that when the noise is not Gaussian, the Kalman filter still provides the best linear state estimator, given only the mean and covariance matrix of noise.

The use of the Kalman filter for data assimilation with realistic ocean models is not straightforward. The most obvious difficulty is the huge dimension ( $n$ ) of the ocean state (of the order of $10^{7}$ ) making the manipulation of the error covariance matrices practically impossible. Several simplified versions of the Kalman filter have been proposed to reduce its computational burden. These sub-optimal Kalman filters basically consist of projecting the state of the system onto a low dimensional subspace (Fukumori and Malanotte-Rizzoli, 1995; Verlaan and Heemink, 1995; Cane et al., 1996). Another difficulty is related to the nonlinear nature of the ocean models. In this case the system equations are generally linearized about the current analysis state leading to the popular, but no longer optimal, extended Kalman (EK) filter (Jazwinski, 1970). However, this amounts to neglect higherorder statistical moments and several studies have shown that it might produce instabilities, even divergence, when implemented with strongly nonlinear systems (Evensen, 1992; Gauthier et al., 1993).

The SEEK filter and its ensemble variant, called SEIK filter, are two alternatives to the Kalman filter, designed for data assimilation with realistic ocean models. These two filters are briefly summarized below. The reader is referred to Pham et al. (1997) and Pham (2001) for a detailed description.

\subsection{The SEEK filter}

The SEEK filter aims at reducing the computational burden of the EK filter by using low rank $(r \ll n)$ matrices approximations of the filter's error covariance matrices. It therefore belongs to the family of square-root Kalman filters (Tippett et al., 2003) and is formulated very alike the reducedrank square root (RRSQRT) EK filter (Verlaan and Heemink, 1995), except for the use of a singular value decomposition (SVD), which is computationally expensive for large values of $r$, at the initial time only. Indeed, the use of low rank error 
covariance matrices allows the decomposition

$P=L U L^{T}$,

where $L$ and $U$ are $n \times r$ and $r \times r$ matrices, respectively. Under this assumption, the algorithm of the EK filter remains mostly unchanged. Only the evolution of the error covariance matrices is avoided and replaced by those of $L$ and $U$. It can then be easily seen that the correction of the EK filter is only applied in the directions parallel to the columns of $L$, hence $L$ will be called "directions of correction" of the filter. Pham et al. (1997) showed that the columns of $L$ converge toward the directions of error growth. This implies that the SEEK filter corrects the forecast in the directions for which the error was not sufficiently attenuated by the dynamics of the model. When the model error is not neglected, the SEEK filter projects this error onto the subspace spanned by the columns of $L$ to avoid continuous increase in the rank of the error covariance matrices, since this would make the filter computationally not affordable. In practice, $r$ can be relatively small (10-100) enabling the implementation of the SEEK filter with high resolution realistic ocean models.

\subsection{The SEIK filter}

Ensemble representations can be efficiently used to represent square-root covariance matrices (Tippett et al., 2003) since the sample covariance matrix of a given ensemble of $N$ members $X^{1}, \ldots, X^{N}$ can be naturally decomposed as in Eq. (1), with $L=1 / \sqrt{N-1}\left[X^{1} \cdots X^{N}\right]$ and $U=I_{N \times N}$. Pham (2001) exploited this feature to develop an ensemble-based variant of the SEEK filter, called SEIK filter. The basic idea of this filter consists of representing the low rank $(r)$ error covariance matrix of the SEEK filter by an ensemble of state vectors using a stochastic approach called the second-order exact sampling scheme (Pham, 2001). Such Monte-Carlo scheme allows to sample the ensemble members so that their mean and sample covariance matrix exactly match the filter's analysis and the corresponding error covariance matrix, respectively. Note that other schemes can also be derived following this approach since the representation (1) is not unique and $P$ can be decomposed as $P=(L \Omega)(L \Omega)^{T}$ for any orthogonal $N \times N$ matrix $\Omega$.

The SEIK filter has strong similarities with the ensemble Kalman (EnK) filter (Evensen, 1994). The main differences between the two filters lies in the procedure of generating the analysis ensemble. The EnK filter is based on perturbed observations (Tippett et al., 2003) in which the analysis ensemble is obtained by assimilating different observations ${ }^{1}$ to each member of the background ensemble. The SEIK filter does not perturb the observations and applies the analysis only once, to provide both the mean analysis and the corresponding error covariance matrix. Then the analysis members are sampled using the second-order exact sampling

\footnotetext{
${ }^{1}$ These observations are created by adding random noise (generated according to the observational error covariance matrix) to the real observations.
}

scheme. The SEIK filter makes also use of the low rank $(r)$ error covariance matrices approximation allowing to use, in a very efficient way, the smallest number of ensemble members, namely $r+1$, thus has an advantage in terms of computational cost with respect to the EnK filter. Furthermore, re-sampling the ensemble every analysis step helps preventing an over-dispersion of the members, which allows the filter to operate more efficiently with a small number of members. Nerger et al. (2005) compared the performances of the EnK and the SEIK filters and found that the SEIK filter generally provides better results, as the perturbed observations approach tends to increase the sampling error of the filter (Lawson and Hansen, 2004).

A schematic illustration of the algorithms of the SEEK and SEIK filters is given in Fig. 1. Starting from the same initialization step, which requires an initial estimate of the model state and a low rank approximation of the corresponding error covariance matrix (generally obtained by applying an empirical functions analysis (EOF) on a set of model outputs), the two filters provide the analysis state in two steps. In the forecast step, the SEIK filter integrates the nonlinear model to evolve the statistics of the forecast while the SEEK filter uses the tangent linear model to do the same work. The analysis step is the same for both filters and it minimizes the error variance of the analyzed estimate in a least-squares sense. The difference in the update formula of the analysis error covariance matrices (more precisely $U$ in the diagram) is only due to the decomposition of the background error covariance matrices. The SEEK and the SEIK filters have therefore the same analysis formula and the only difference between them is the use of the tangent linear model or of a nonlinear ensemble forecasting scheme to perform the evolution of the statistics of the forecast state. Using the same rank for the error covariance matrices in the two filters, allows therefore to efficiently assess the relevance of both approaches on the assimilation results by comparing the performances of the filters with high resolution primitive equations models. It is important to note that under this condition the computational cost of both filters is basically the same.

\section{The ocean models}

The model used in the assimilation experiments is the Princeton Ocean model (POM) implemented in two (low and high) horizontal resolution cases as stated before. POM has been previously used in numerous applications like the Adriatic Sea (Zavatarelli and Pinardi, 1995), the Mediterranean Sea (Drakopoulos and Lascaratos, 1997), and the Levantine Sea (Korres and Lascaratos, 2003).

\subsection{The POM model}

POM is a primitive equations finite difference model which makes use of the hydrostatic and Boussinesq approximations. The model solves the 3-D Navier-Stokes equations on an Arakawa-C grid with a numerical scheme that con- 
SEEK Filter

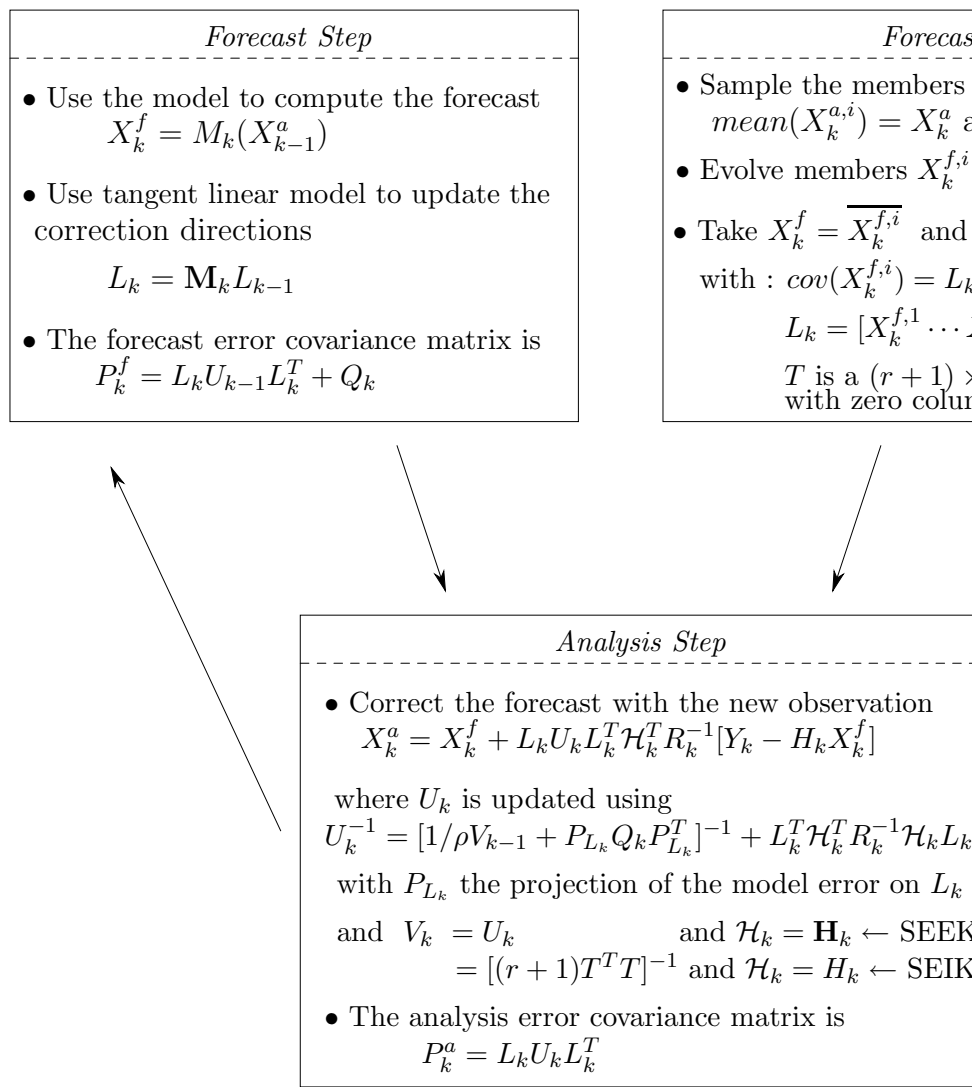

SEIK Filter

recast Step

$X_{k}^{a, i}$ such as

$\operatorname{cov}\left(X_{k}^{a, i}\right)=P_{k}^{a}$

$=M_{k}\left(X_{k}^{a, i}\right)$

$P_{k}^{f}=\operatorname{Cov}\left(X_{k}^{f, i}\right)+Q_{k}$

$\left[(r+1) T^{T} T\right]^{-1} L_{k}^{T}$

$\left.X_{k}^{f, r+1}\right] \cdot T$

sums

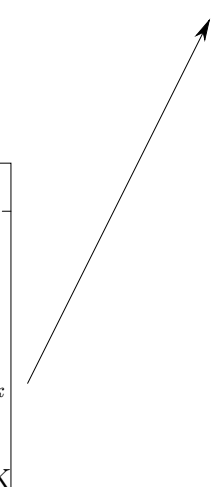

Fig. 1. A diagram summarizing the algorithms of the SEEK and SEIK filters: $X^{a}$ and $X^{f}$ denote the analysis and forecast states, $M$, M, $H$ and $\mathbf{H}$ the transition and the observation operators and their linearized operators, $Q$ and $R$ the corresponding model and observation errors covariance matrices, and $\rho$ is a forgetting factor. Note that the computation of the analysis and forecast error covariance matrices $P^{a}$ and $P^{f}$ is not needed for the filters algorithms. They have been included for completeness.

serves mass and energy. The spatial differences are central and explicit in the horizontal and central and implicit in the vertical. Centered differences are used for the time integration (leapfrog scheme) of the primitive equations. In addition, since the leapfrog scheme has a tendency for the solution to split at odd and even time steps, an Asselin filter is used at every time step. The numerical computation is split into an external barotropic mode with a short time step (dictated by the CFL condition) solving for the time evolution of the free surface elevation and the depth averaged velocities, and an internal baroclinic mode which solves for the vertical velocity shear. Horizontal mixing in the model is parameterized according to Smagorinsky (1963) while vertical mixing is calculated through the Mellor and Yamada 2.5 turbulence closure scheme. The reader is referred to Blumberg and Mellor (1987) for a detailed description of POM.

The model state vector is composed of all prognostic (state) variables of the model at each sea grid point. The state variables consist of the sea surface elevation, the zonal and meridional components of velocity, potential temperature, salinity, the turbulent kinetic energy and the turbulent kinetic energy times the turbulent length scale.

\subsection{The areas of POM implementation}

Two configurations of POM were considered to test the performance of the SEEK and SEIK filters: (i) low horizontal resolution model $1 / 4^{\circ} \times 1 / 4^{\circ}$ of the Mediterranean Sea, and (ii) high horizontal resolution $1 / 100^{\circ} \times 1 / 100^{\circ}$ of the Pagasitikos Gulf (a coastal area within the Aegean Sea), each with 25 sigma levels in the vertical logarithmically distributed near the surface and the bottom. A small domain (Pagasitikos Gulf) was considered for the high resolution case to save computational time. The general behavior of the filters is not expected to be sensitive to the size of the domain. The model bathymetry was obtained from the US Navy Digital Bathymetric Data Bases DBDB5 and DBDB1 and is shown in Fig. 2. The Mediterranean model covers the geographical area $7^{\circ} \mathrm{W}$ to $36^{\circ} \mathrm{E}$ and $30^{\circ} \mathrm{N}$ to $46^{\circ} \mathrm{N}$ and has one open boundary located at $7^{\circ} \mathrm{W}$. The Pagasitikos Gulf model covers the area $22.8^{\circ} \mathrm{E}$ to $23.3^{\circ} \mathrm{E}$ and $39^{\circ} \mathrm{N}$ to $39.4^{\circ} \mathrm{N}$ and has two open boundaries to the south and east respectively. The number of grid points was therefore $175 \times 65$ for the Mediterranean model and $49 \times 49$ for the Pagasitikos Gulf model. Open boundary conditions were set as follows: 

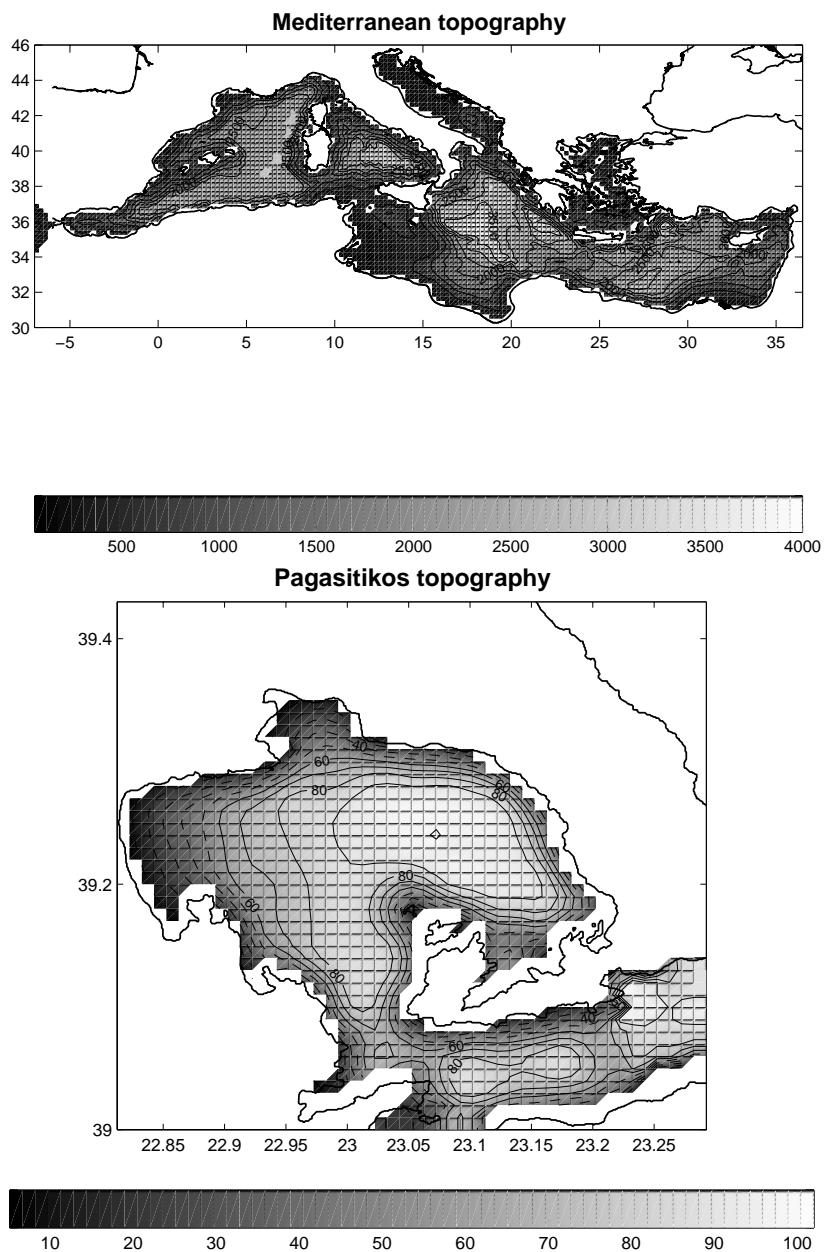

Fig. 2. Topography of the Mediterranean (upper panel) and the Pagasitikos (lower panel) models.

- Zero gradient condition for the free surface elevation.

- Flather (1976) boundary condition for the barotropic velocity normal to the open boundary.

- Sommerfeld radiation for the internal (baroclinic) velocities.

- Temperature and salinity at the open boundaries are advected upstream. When there is inflow through the open boundary, these fields are prescribed from the MODBMED4 seasonal climatology.

In order to adjust the model dynamics and achieve a perpetually repeated seasonal cycle before applying the interannual atmospheric forcing, the Mediterranean and Pagasitikos models were integrated climatologically for 19 years and 10 years, respectively. The Mediterranean model climatological run was initialized with the MODB-MED4 spring temperature and salinity profiles and the initial velocities were set to zero. For the Pagasitikos Gulf model the above data set was enriched with CTD data acquired through the research project Development of an Integrated Policy for the Sustainable Management of Pagasitikos Gulf. The surface
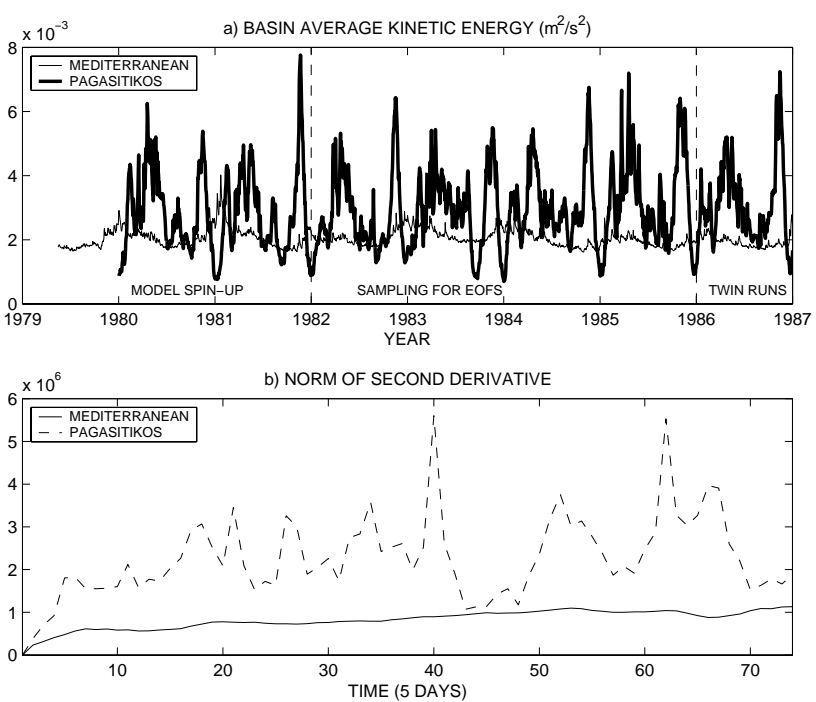

Fig. 3. Basin average kinetic energy and norm of the second derivative for both models.

forcing fields (monthly climatological wind stresses, upward heat flux, net shortwave radiation and evaporation rates) were derived from the 1979-1993 ECMWF $1^{\circ} \times 1^{\circ}$ reanalysis 6-h atmospheric data set, except for the precipitation fields which were derived from Jaeger monthly climatology. The reference experiment to which all assimilation runs refer, was initialized from the end of the 19th year and 10th year of climatological integration for the Mediterranean and Pagasitikos cases, respectively. These experiments were forced with the 1979-1994 ECMWF reanalysis 6-h atmospheric data (wind velocity, air temperature and relative humidity) and cloud cover and precipitation data taken from COADS monthly $1^{\circ} \times 1^{\circ}$ fields for the same period. Proper bulk formulae were used to compute the surface momentum, heat and freshwater fluxes at each time step of model integration taking into account the SST predicted by the model itself.

The evolution of the basin average kinetic energy within the time period 1979-1987 for the two models is shown in Fig. 3a. The low resolution Mediterranean model involves a strong seasonal cycle to which inter-annual anomalies are superimposed with the most important being that of year 1981 forced by the corresponding wind stress anomalies. The kinetic energy of the Pagasitikos model on the other hand is largely affected by the activity and the instabilities of a large mesoscale eddy that on average is developed in the central part of the Pagasitikos basin and changes polarity and shape according to the momentum and buoyancy fluxes at the surface of the basin. Thus the kinetic energy of the basin consists of a seasonal signal to which quasi-chaotic mesoscale fluctuations are dominant, suggesting stronger nonlinear behavior of the high resolution model.

The nonlinearities in the two models were also assessed by checking the norm of the second derivative of the model solution with respect to the initial conditions. The second derivative was computed via finite differencing. First, a domain 

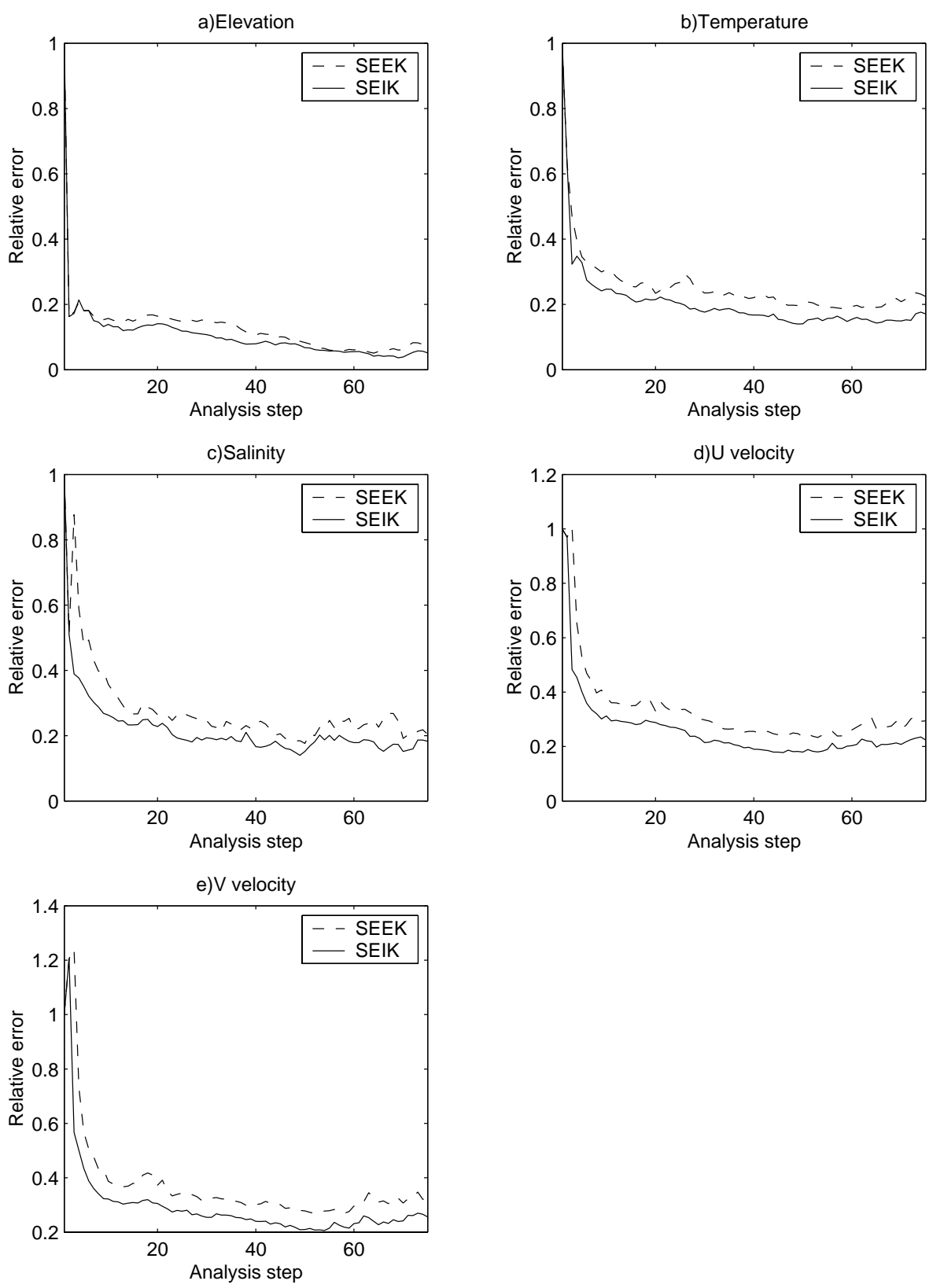

Fig. 4. Evolution of the RRMS error as function of time for the SEEK and SEIK filters with the low resolution Mediterranean model using an error covariance matrix of rank 50 (analysis step is 5 days).

scale perturbation ( $1 \%$ of the model state vector standard deviation as deduced from the 4-year ensemble) was added to the initial conditions. The second derivative with respect to the initial conditions (which can be used as a measure of the degree of nonlinearity in the model) was obtained by adding the positively and the negatively perturbed runs and subtracting twice the unperturbed run. The norm of the second derivative for the Mediterranean and Pagasitikos models is shown in Fig. $3 b$ at intervals of 5 days. In both cases, the major contribution to the second derivative norm is due to the barotropic velocity field and the associated free surface elevation. The nonlinear variations in the low resolution Mediterranean model are shown to be less important while the presence of strong eddy activity in the Pagasitikos model and the flow exchange with the open boundaries makes this model highly nonlinear.

\section{Experiments}

\subsection{Experiments setup}

The initialization of the filters requires an initial state estimate and a low rank approximation of the corresponding error covariance matrix. A common strategy for the estimation of the error covariance matrix is to use model statistics as an approximation of the true system statistics (Pham 
et al., 1997). Then by appropriate sampling of model state vectors one can obtain an approximation of the filter's covariance matrix through the dominant empirical orthogonal functions (EOFs). In the twin experiments setup, the models were first integrated for a 2-year period (1980-1981) in order to achieve a quasi adjustment of the model climatological dynamics to the ECMWF inter-annual forcing. Next, another integration of 4 years (1982-1985) was carried out to generate a historical sequence of model states sampled every 2 days. Since the state variables are of different nature, a multivariate EOF analysis was applied on the sampled set of 730 state vectors. In this analysis, model state variables were normalized by the inverse of the square-root of their domain-averaged variances.

A reference model run was first carried out and a sequence of 73 state vectors was retained every 5 days during a oneyear period over 1986. These reference states, considered as the "true states" of the sea, were retained to be latter compared with the fields produced by the filters. The assimilation experiments were performed during the same period, using pseudo-observations of sea surface height for the Mediterranean model, and temperature and salinity profiles for the Pagasitikos model (according to the available observation systems in each domain) which were extracted every 5 days and every 4 grid points from the reference states. Independent Gaussian errors of mean zero and standard deviation equal to $5 \%$ of the observed standard deviation (as computed from the model SSH during 1982-1985) were added to the pseudo-observations. The filters were initialized from the mean state vector of the 4-year period (1982-1985) used for the calculation of the multivariate EOFs. In all the assimilation experiments, the model was assumed perfect $\left(Q_{k}=0\right)$. Another experiment is initialized from the same mean state vector, as above, and is integrated over 1986 without any assimilation. This is the model pure forecast (free-run without assimilation).

To avoid Kalman filters divergence problems due to the underestimation of the error covariance matrices (by low rank matrices) and/or to reduce the influence of model errors when real data are assimilated, the inverse of the prior covariance matrices were artificially multiplied by a factor, called forgetting factor (Pham, 2001), of 0.8 in all the assimilation runs.

The performance of the filters was evaluated by comparing the filter's analysis errors, for the whole state vector or separately for each model state variables, relative to the pure forecast error (free-run without assimilation)

$R R M S=\sqrt{\frac{\int\left(X^{\text {analysis }}-X^{\text {true }}\right)^{2}}{\int\left(X^{\text {free run }}-X^{\text {true }}\right)^{2}}}$.

4.2 Assimilation results

\subsubsection{SEEK versus SEIK with the low resolution model}

\section{Twin Experiments}

The SEEK and SEIK filters were first implemented under
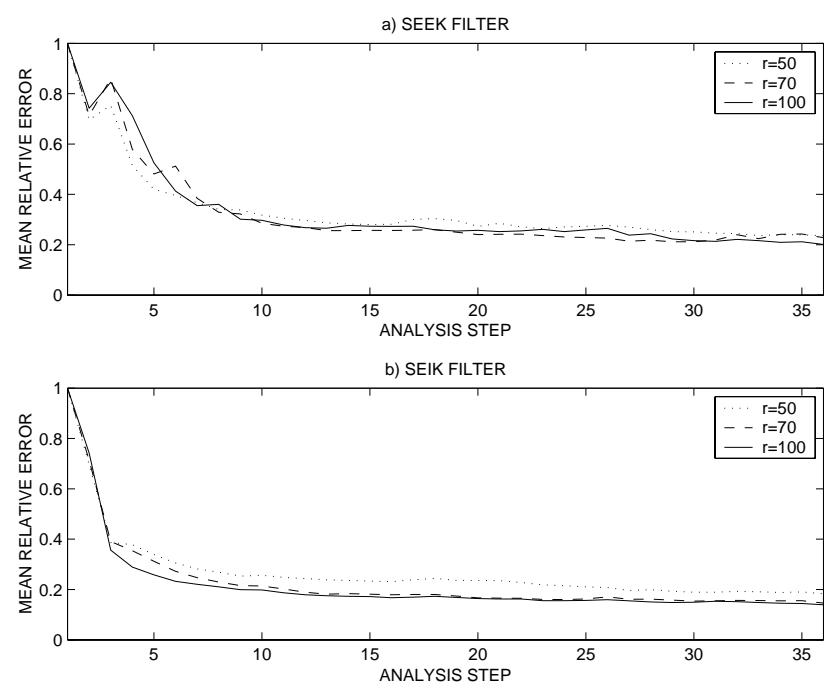

Fig. 5. Evolution of the RRMS error as function of time for the SEEK and SEIK filters with the low resolution Mediterranean model using different ranks for the error covariance matrices (analysis step is 5 days).

the same conditions using an error covariance matrix of rank 50. The evolution of the RRMS for both runs as a function of time is plotted in Fig. 4. It can be seen that a large reduction of the estimation error is achieved at the first analysis step. Subsequent analyzes are significantly less important and both filters are able to reduce the analysis error up to a certain level. Although the SEEK filter is performing efficiently with the low resolution model, the use of nonlinear ensemble forecasting in the SEIK filter is more stable and provides better assimilation results for all model state variables.

\section{Sensitivity to the rank of the covariance matrices}

Several assimilation runs were performed to study the sensitivity of the SEEK and SEIK filters with respect to the rank of the error covariance matrix. Figure 5 shows the filters RRMS for the whole state vector using 50, 70 and 100 directions of correction. The use of additional modes is shown to improve the overall performance of the SEEK filter, but this is only true up to a certain value. Indeed, the assimilation results obtained with this filter saturate, or even sometimes degrade, when more than 70 directions of correction were used. This observation is consistent with that reported by Verron et al. (1998) and Hoteit and Pham (2003) and suggests that the evolution of the last EOFs, which generally represent fine scale variations is not well supported by the tangent linear model used in the SEEK filter. It is therefore more beneficial in certain situations to keep these modes out of the correction basis of the filter. By contrast, although the SEIK filter is showing some degree of saturation, it is clear that its behavior is progressively improved when more ensemble members are used. This can be explained by the fact that at each analysis step, the 


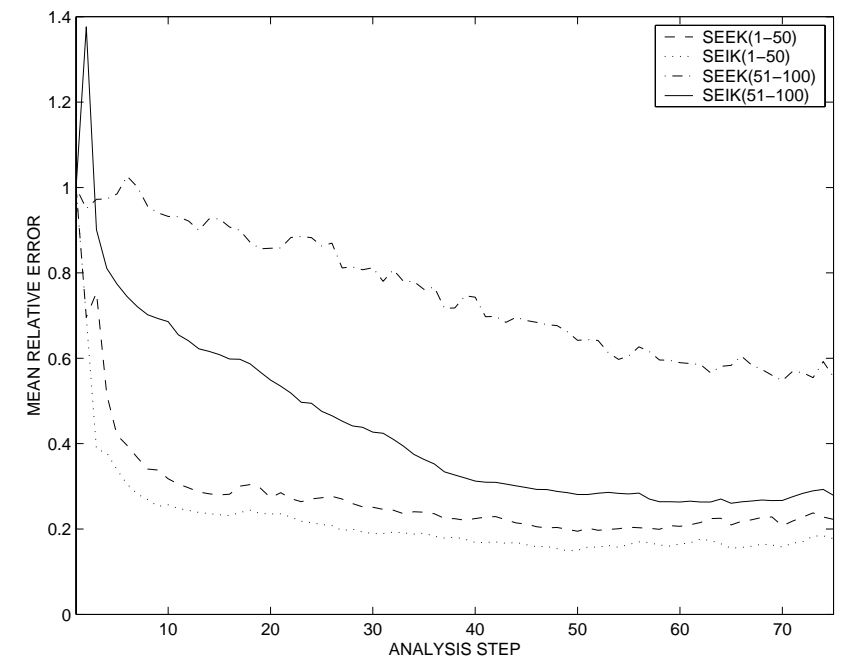

Fig. 6. Evolution of the averaged RRMS error of all state variables as function of time for the SEEK and SEIK filters with the low resolution Mediterranean model using the last 50 EOFs to initialize the filters correction directions (analysis step is 5 days).

SEIK filter evolves combinations of modes weighted by their relevance (i.e. estimated variance). Such combinations statistically attenuate the "bad influence" of fine scale variations in the forecast ensemble, and therefore provide a better estimate of the filter's directions of correction.

\section{Sensitivity to the initial error covariance matrix}

Another sensitivity experiment was conducted to assess the influence of using "bad initial directions of corrections" on the convergence of the SEEK and SEIK filters. To this matter, we ran the two filters using the 51-100 EOFs as initial directions of correction and compared them to the original runs (with the first 1-50 EOFs as initial directions of correction). The RRMS of these runs are plotted in Fig. 6 . The convergence of the SEEK filter is shown to be strongly slowed down when the initial directions of correction are not well estimated. The error decreasing is also more unstable. Except for the second correction, which can be explained by the widely spread initial forecast ensemble members, the SEIK filter seems to be much less affected by the use of 'bad' EOFs. This suggests that the nonlinear ensemble forecasting is more robust with respect to the initial conditions and it guides the Kalman filter toward the directions of error growth of the model much faster than the tangent linear model.

\section{Assimilation of AVISO altimetry data}

Another demonstration of the performance of the SEEK and SEIK filters is given by assimilating real merged T/P and ERS sea level anomalies (SLA) on a weekly basis for one-year period (1993). The merged altimetric data were processed by the AVISO altimetry group mapped on a $1 / 3^{\circ} \times 1 / 3^{\circ}$ Mercator grid using a space-time objective anal-
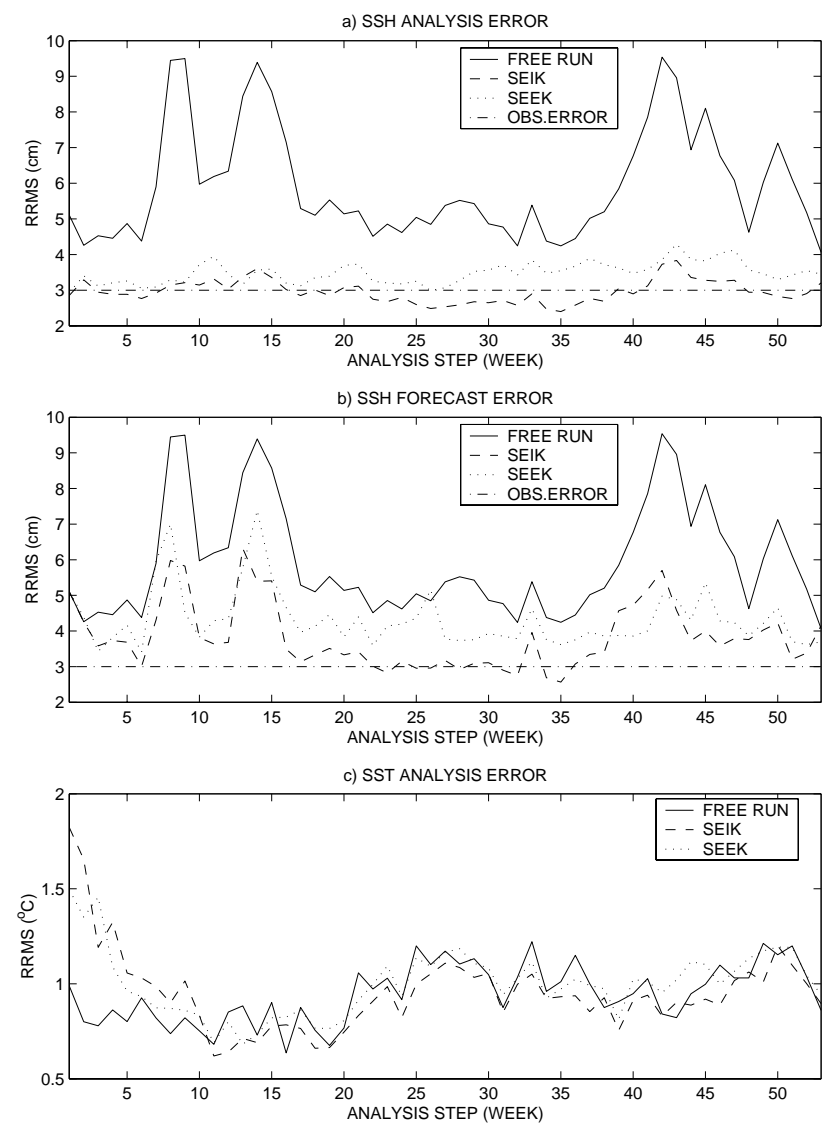

Fig. 7. Evolution of the RMS error as function of time for the SEEK and SEIK filters with the low resolution Mediterranean model assimilating real merged Topex and ERS SSH observations.

ysis method that accounts for long wavelength errors (Le Traon et al., 1998). To assimilate the AVISO anomalies, a mean sea surface height ( $\mathrm{SSH}$ ) needs to be added so that they can be compared with the filter's forecast SSH. Following Verron et al. (1998), we added a mean SSH obtained from the model's free-run over 4-year period (1989-1992), used for the calculation of the EOFs. The problem of the mean SSH estimation, not addressed here, remains open for future studies. Hindcast runs using the SEEK and SEIK filters were performed to compare the performances of the two filters in a near real-time situation by assimilating AVISO altimetry data on a weekly basis. The model has been also ran without assimilation but starting from the initial conditions of the filters to assess the relevance of the assimilation schemes. Guided by the sensitivity studies results, an error covariance matrix of rank 50 was used to save computational time. In all runs, the observational error was set to $3 \mathrm{~cm}$. The performance of the filters is evaluated by monitoring the basin average misfit (RMS) between the filters' estimates (forecast and analysis) and observed SSH, as well as with Reynolds $1 / 4^{\circ} \times 1 / 4^{\circ}$ SST data. The latter are independent data (not assimilated) and they are used to assess the consistency of the filter analysis with the model dynamics following a cross-validation approach. 
RMS differences of the AVISO SSH with the free-run solution and the filters analysis are shown in Fig. 7a. The model free-run presents an average RMS difference of $6 \mathrm{~cm}$ with peaks reaching approximately $9.5 \mathrm{~cm}$. The SEEK filter efficiently reduces the average RMS difference by $2.5 \mathrm{~cm}$ but generally its solution does not fit the data within the specified observational errors $(3 \mathrm{~cm})$. The SEIK filter is showing a better behavior and is able to reduce the model/data difference to a lower level than $3 \mathrm{~cm}$, which is a strong indication of the relevance of this filter. The same RMS differences but for the forecast/data are plotted in Fig. 7b. This is the quantity that one should monitor to make sure that the filter analysis is consistent with the model dynamics. It can be seen that the model tends to drift from the data after each forecast step, especially when the model forecast error is high. Both filters analysis successfully improves the forecast with the new observations and again the SEIK filter provides a better solution. Finally, one can see from Fig. 7c that the impact of the assimilation on the independent SST data is dramatically less pronounced, with the SEIK filter showing a better behavior. This comparison also confirms the analysis/model consistency. The SST field is even shown to be better simulated by the model run without assimilation during the first 10 weeks, but this can be attributed to the time needed by the filters to update their internal statistics. The poor performance of the filters with the SST is probably associated with the AVISO climatology problem and more data need to be assimilated for a better control of the model SST. The lack of adequate representation of the model imperfections may also be another reason. Overall, the results of this hindcast experiment are consistent with those obtained in the twin experiments approach: both filters were able to improve the consistency between the model and the data but the SEIK filter generally provides a better solution than the SEEK filter.

\subsubsection{SEEK versus SEIK with the high resolution model}

The SEEK and SEIK filters, with the same configuration as described in the twin experiments of the low resolution case, were used to assimilate data into the high resolution Pagasitikos model. Figure 8 compares the temporal evolution of the RRMS obtained by each filter. After a good first correction, the overall behavior of the SEEK filter is strongly unstable with a tendency of an increase in the analysis error over time. This suggests that the use of the linearized model to perform the evolution of the forecast distribution might be inadequate for the high resolution ocean model because of the presence of strongly nonlinear fine-scale variations. The use of nonlinear ensemble forecasting seems to be more appropriate for this problem. Indeed, the SEIK filter is more stable and provides better estimates of the model states than the SEEK filter. However, looking more closely to the assimilation results, one can see that the SEIK filter is also showing some weaknesses toward the end of the assimilation window with the RRMS increasing at a closely similar rate to the SEEK filter, despite the noticeable overall improvement with respect to the latter. This is probably due to the linear analysis step of the SEIK filter which, as in the SEEK filter, only uses the Gaussian part of the forecast distribution.

Finally, in an attempt to improve the performance of the SEEK filter, several experiments were performed to investigate if the use of more observations over shorter assimilation steps enhances the performance of the SEEK filter. This can be expected since the linearization of the model will be carried out over shorter periods where the model behavior is more linear, and therefore the assumption of the tangent linear model is more supported. The results of these experiments (not presented here) actually show some improvements. However, small linearization errors still continue to propagate in time developing instabilities in the filter's behavior. The overall improvements were therefore practically insignificant with respect to the huge amount of assimilated data. Since these data will never be available in real situations, the use of the tangent linear model seems to be questionable for the strongly nonlinear high resolution model.

\section{Discussion}

Kalman filtering is one of the most promising tools for data assimilation in oceanography. Since the Kalman filter is only optimal for linear models, two different approaches are generally used for the implementation of this estimation technique to nonlinear models. The first approach consists of linearizing the model equations about the current analysis leading to the so-called extended Kalman filter. The other approach is based on the use of nonlinear Monte-Carlo ensemble forecasting methods to represent estimation errors with an ensemble of state vectors. Until recently, both techniques were shown to perform fairly well when applied to relatively low resolution models. In this paper, the efficiency of these two approaches was assessed with the implementation of two advanced Kalman filters, the SEEK and the SEIK, with a low and a high resolution realistic primitive equations ocean models. Both filters are based on a low-rank approximation of the error covariance matrix and differ only in the use of linearization (SEEK) or a Monte Carlo technique (SEIK) to overcome model nonlinearities. As expected, numerical experiments show that the tangent linear model can be efficiently used to update the estimation error in time when the problem of data assimilation is considered with the low resolution model, although its performance never equals that of the ensemble method. The advantage of using the ensemblebased approach was however significantly more pronounced when the filters were implemented with the strongly nonlinear high resolution model. This can be explained by the ability of the ensemble approach to account for higher order terms in the Taylor expansion of the model transition operator than the tangent linear model. Additionally, the latter approach was also shown to be less sensitive with respect to the initial conditions and to the approximated rank of the error covariance matrices. Another advantage (not explored here) of the SEIK filter over the SEEK filter is the possibility of stochastically including the model error to the ensemble 

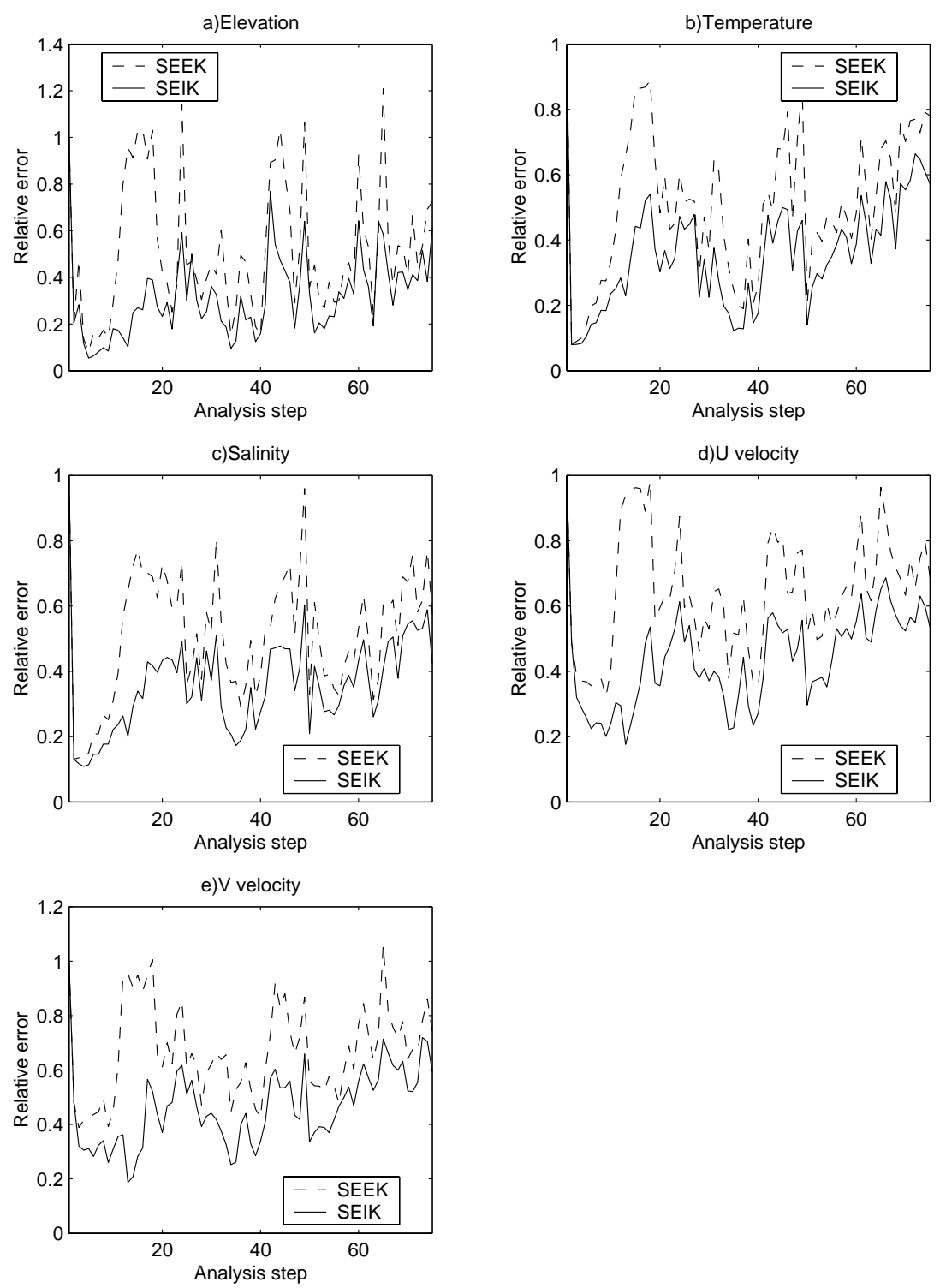

Fig. 8. Evolution of the RRMS error as function of time for the SEEK and SEIK filters with the high resolution Pagasitikos model (analysis step is 5 days).

members as in the EnK filter. Considering that the computational cost of both approaches is almost the same, the results of this study support the use of the ensemble nonlinear forecasting techniques with high resolution primitive equation ocean models.

Ensemble-based Kalman methods are definitely more robust with model nonlinearities. Their simplicity and more importantly their affordable computational burden make them well suited for the problem of oceanic data assimilation. But even these methods, which were shown so far to be the most promising approach to deal with this problem, can still be improved. Indeed, their linear analysis formulae are still based on the assumption of prior error Gaussian distributions as in the original Kalman filter. The optimal nonlinear filter Pham (2001), which resolves the fully nonlinear Bayesian approach for the state estimation problem, is a serious alternative for the ensemble Kalman filter methods. But since the numerical implementation of the algorithm of this filter is very demanding in computing power, some simplifications will be inevitable for realistic ocean applications. A work in this sense is currently under investigation.

Acknowledgements. This work has been partially supported by the MFSTEP and FERRYBOX EU research projects. Reynolds SST data were provided by the NOAA-CIRES Climate Diagnostics Center, Boulder, Colorado, USA. The SLA products were supplied by the CLS Space Oceanography Division, Toulouse, France. The authors would like to thank A. Eleftheriou for his constructive criticism during the preparation of this work and L. Nerger, B. Cornuelle and I. Fukumori for valuable discussions and exchange of information.

Edited by: O. Talagrand

Reviewed by: two referees 


\section{References}

Blumberg, A. F. and Mellor, G. L.: A description of a three-dimensional coastal ocean circulation model, in: ThreeDimensional Coastal Ocean Circulation Models, edited by: Heaps, N. S., AGU, Washington, D.C., Coastal Estuarine Sci., 4, 1-16, 1987.

Cane, M. A., Kaplan, A., Miller, R. N., Tang, B., Hackert, E. C., and Busalacchi, A. J.: Mapping tropical Pacific sea level: data assimilation via a reduced state Kalman filter, J. Geophys. Res., 101, C10, 22 599-22 617, 1996.

Drakopoulos, P. G. and Lascaratos, A.: Modeling the Mediterranean Sea: climatological forcing, J. Mar. Sys., 20, 157-173, 1997.

Evensen, G.: Sequential data assimilation with a nonlinear quasigeostrophic model using Monte Carlo methods to forecast error statistics, J. Geophys. Res., 99, C5, 10 143-10 162, 1994.

Evensen, G.: Using the extended Kalman filter with a multilayer quasi-geostrophic ocean model, J. Geophys. Res., 97, C11, 17 905-17 924, 1992.

Flather, R. A.: A tidal model of the northwest European continental shelf, Mem. Soc. R. Sci. Liege Ser., 6, 10, 141-164, 1976.

Fukumori, I. and Malanotte-Rizzoli, P.: An approximate Kalman filter for ocean data assimilation: an example with an idealized gulf stream model, J. Geophys. Res., 100, C4, 6777-6793, 1995.

Gauthier, P., Courtier, P., and Moll, P.: Assimilation of simulated wind Lidar data with a Kalman filter, Mon. Wea. Rev., 121, 1803-1820, 1993.

Ghil, M. and Malanotte-Rizzoli, P.: Data assimilation in meteorology and oceanography, Adv. Geophys., 33, 141-266, 1991.

Hoteit, I. and Pham, D. T.: Evolution of the reduced state space and data assimilation schemes based on the Kalman filter, J. Meteo. Soc. Japan, 81, 21-39, 2003.

Jazwinski, A. H.: Stochastic processes and filtering theory, Academic Press, New York, 376, 1970.

Kalman, R. E.: A new approach to linear filtering and prediction problems, Trans. ASME Ser. D, J. Basic Eng., 82D, 35-45, 1960.

Köhl, A. and Willebrand, J.: An adjoint method for the assimilation of statistical characteristics into eddy-resolving ocean models, Tellus, 54, 4, 406-425, 2002.

Korres, G. and Lascaratos, A.: An eddy resolving model of the Aegean and Levantine basins for the Mediterranean Forecasting System Pilot Project (MFSPP): Implementation and climatological runs, Ann. Geophys., 21, 205-220, 2003,

\section{SRef-ID: 1432-0576/ag/2003-21-205.}

Lawson, G. and Hansen, J.: Implications of stochastic and deterministic filters as ensemble-based data assimilation methods in varying regimes of error growth, Mon. Wea. Rev., 132, 8, 19661981, 2004
Le Traon P. Y., Nadal, F., and Ducet, N.: An improved mapping method of multi-satellite altimeter data, J. Atmos. Ocean. Tech., 25, 522-534, 1998.

Madsen, H. and Canizares, R.: Comparison of extended and ensemble Kalman filters for data assimilation in coastal area modelling, Int. J. Num. Meth. Fluids, 31, 961-981, 1999.

Miller, R. N., Ghil, M., and Gauthiez, F.: Advanced data assimilation in strongly nonlinear dynamical systems, J. Atmos. Sci., 51, 8, 1037-1056, 1994.

Nerger, L., Hiller, W., and Schröter, J.: A Comparison of Error Subspace Kalman Filters, part 1: Filter algorithms, Evaluation of Numerical Experiments, http://www.awi-bremerhaven. de/Publications/Ner2003c.pdf, Tellus, accepted, 2005.

Pham, D. T.: Stochastic Methods for sequential data assimilation in strongly nonlinear systems, Mon. Wea. Rev., 129, 1194-1207, 2001.

Pham, D. T., Verron, J., and Roubaud, M. C.: Singular evolutive Kalman filter with EOF initialization for data assimilation in oceanography, J. Mar. Syst., 16, 323-340, 1997.

Smagorinsky, J.: General circulation experiments with the primitive equations, I. The basic experiment, Mon. Weather Rev., 91, 99164, 1963.

Tippett, M., Anderson, J., Bishop, C., Hamill, T., and Whitaker, J.: Ensemble square root filters, Mon. Wea. Rev., 131, 1485-1490, 2003.

Verlaan, M. and Heemink, A. W.: Reduced rank square root filters for large scale data assimilation problem, Second International Symposium on Assimilation of Observations in Meteorology and Oceanography, WMO, 247-252, 1995.

Verron, J., Gourdeau, L., Pham, D. T., Murtugudde, R., and Busalacchi, A. J.: An extended Kalman filter to assimilate satellite altimeter data into a nonlinear numerical model of the tropical pacific: method and validation, J. Geophys. Res., 104, C3, 5441-5458, 1998.

Zang, X. and Malanotte-Rizzoli, P.: A comparison of assimilation results from the ensemble Kalman filter and a reduced-rank extended Kalman filter, Nonlin. Processes Geophys., 10, 477-491, 2003,

SRef-ID: 1607-7946/npg/2003-10-477.

Zavatarelli, M. and Pinardi, N.: The Adriatic Sea general circulation: modeling with the Princeton Ocean Model, Ann. Geophys., 13, (suppl. 2), 1995. 